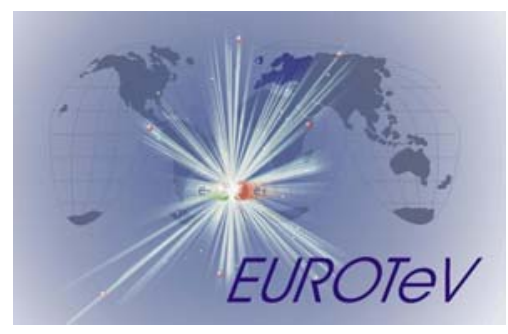

\title{
Vacuum Systems for the ILC Helical Undulator
}

\author{
O.B. Malyshev ${ }^{1,2}$, D.J. Scott ${ }^{1,2}$, I.R. Bailey ${ }^{3,2}$, D.P. Barber ${ }^{4}$, E. Baynham ${ }^{5}$,
} T. Bradshaw ${ }^{5}$, A. Brummitt ${ }^{5}$, S. Carr ${ }^{5}$, J.A. Clarke ${ }^{1,2}$, P. Cooke ${ }^{3}$, J.B. Dainton ${ }^{2,3}$, Y. Ivanyushenkov ${ }^{5}$, L.I. Malysheva ${ }^{2,3}$, G.A. Moortgat-Pick ${ }^{6}$, J. Rochford $^{5}$

March 26, 2007

\begin{abstract}
The International Linear Collider (ILC) positron source uses a helical undulator to generate polarised photons of $\sim 10 \mathrm{MeV}$ at the first harmonic. Unlike many undulators used in synchrotron radiation sources, the ILC helical undulator vacuum chamber will be bombarded by photons, generated by undulator, with energies mostly below that of the $1^{\text {st }}$ harmonic. Achieving the vacuum specification, of $\sim 100 \mathrm{nTorr}$, in a narrow chamber, of 4 to $6 \mathrm{~mm}$ inner diameter, with a long length, of $100-200 \mathrm{~m}$, makes the design of the vacuum system challenging. This paper describes the vacuum specifications, calculations of the flux and energy of photons irradiating the undulator vacuum chamber and considers possible vacuum system design solutions for two cases: cryogenic and room temperature.
\end{abstract}

\footnotetext{
${ }^{1}$ ASTeC, Daresbury Laboratory, Warrington, WA4 4AD,England

${ }^{2}$ Cockcroft Institute, Warrington, WA4 4AD,England

${ }^{3}$ Department of Physics, University of Liverpool, Oxford St., Liverpool, L69 7ZE, UK

${ }^{4}$ DESY-Hamburg, Notkestraße 85, 22607 Hamburg, Germany

${ }^{5}$ CCLRC Rutherford Appleton Laboratory, Chilton, Didcot, Oxfordshire OX11 0QX, UK

${ }^{6}$ Institute of Particle Physics Phenomenology, University of Durham, Durham DH1 3LE, UK, and CERN, CH-

1211 Genève 23, Switzerland
} 


\section{Introduction}

In the baseline design the positrons for the International Linear Collider are produced via pair production in a metallic target hit by a photon drive beam. The photons are produced using a helical undulator and the main ILC electron beam at $150 \mathrm{GeV}$. The length of the undulator is $\sim 100 \mathrm{~m}$ however for a polarised positron beam there needs to be approximately $200 \mathrm{~m}$ of active undulator with a peak field of $\sim 0.8 \mathrm{~T}$.

To provide such a strong magnetic field with short periods of $\sim 12 \mathrm{~mm}$ the aperture available for the beam and vacuum chamber is very small. The most realistic vacuum chamber inner diameter (ID) will be in the range of 4 to $6 \mathrm{~mm}$. The traditional vacuum technologies for narrow gap undulators could not be used here: ante-chamber design could be used only in a planar undulator; in-vacuo design does not provide sufficient vacuum conductance from the beam axis to the pump because the gap size between magnet blocks is too small and length of continuous undulator is too large.

\section{Sources of Gas in a Vacuum System}

To perform a vacuum design of the helical undulator the sources of gas should first be identified.

\subsection{Thermal stimulated desorption}

All materials placed in vacuum desorb molecules of gas and are sources of gas in a vacuum chamber. The desorption rate depends on many factors including, the material, its cleaning, treatment and pre-history. Thermal desorption rates for stainless steel, well-known as a good vacuum material, can reach the level of $10^{-12} \mathrm{mbar} \cdot 1 /\left(\mathrm{s} \cdot \mathrm{cm}^{2}\right)$ after $24 \mathrm{hrs}$ bake-out at $300^{\circ} \mathrm{C}$ and weeks of pumping. 


\subsection{Photon stimulated desorption}

An effect, hitherto generally unconsidered, now needs to be included due to the unusually long length of the ILC helical undulator: some non-negligible fraction of photons generated by the undulator will irradiate the vacuum chamber walls.

\subsubsection{PSD yield as a function of photon dose}

Photodesorption yields, $\eta$ (molecules/photon), depend on the material, cleaning, treatment, pre-history and accumulated photon dose. There are a number of experimental results published in the literature for photodesorption yields, $\eta$, measured as function of accumulated photon dose, D, for different materials and measured up to certain photon doses (for example, see $[1,2,3])$. Schematically, a typical behaviour of the desorption yield as a function of photon dose is shown in Figure 1. Initially, the photodesorption yield might be almost independent (for instance, stainless steel after bakeout at $300^{\circ} \mathrm{C}$ for $24 \mathrm{hrs}$ with doses up to $\sim 10^{21}$ photons $/ \mathrm{m}$ ) or having very low dependence on photon dose (as copper and aluminium) then, for longer irradiation time, the photodesorption yield at room temperature can be described as a function of accumulated photon dose as:

$$
\eta=\eta_{0}\left(\frac{D_{0}}{D}\right)^{\alpha}, \quad 0.65<\alpha<1
$$

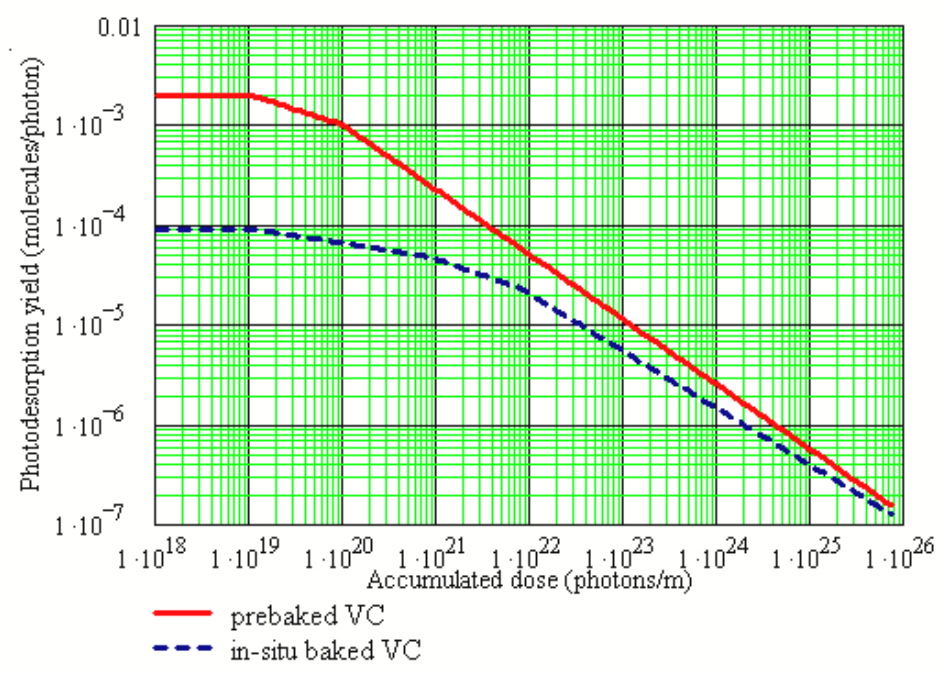

Figure 1. PSD yield for $\mathrm{CO}$ for pre-baked and in-situ baked stainless steel vacuum chambers. Yields for doses higher then $10^{23}$ photons/m are extrapolations. 


\subsubsection{PSD yield as a function of vacuum chamber temperature}

The PSD yields from the vacuum chamber wall also depends upon the wall temperature:

- Initial desorption at $77 \mathrm{~K}$ is an order of magnitude lower than the same material at room temperature, but its conditioning takes longer [4]:

$$
\eta(77 K)=\eta_{0}(77 K)\left(\frac{D_{0}}{D}\right)^{\alpha}, \quad \alpha \approx 0.3
$$

- Initial desorption at $4.2 \mathrm{~K}$ is about two orders of magnitude lower than the same material at room temperature, but its conditioning goes very slow [4,5]:

$$
\eta(4 K)=\eta_{0}(4 K)\left(\frac{D_{0}}{D}\right)^{\alpha}, \quad \alpha \approx 0.1
$$

\subsubsection{PSD yield as a function of photon critical energy}

The photon desorption also depends on photon energy. Studies have shown that the PSD yield changes with photon critical energy such that it is:

- proportional to the critical photon energy $\varepsilon_{\mathrm{c}}$ when $\varepsilon_{\mathrm{c}}<1 \mathrm{keV}$ [6];

- weak increase on the critical photon energy $\varepsilon_{\mathrm{c}}$ when $1 \mathrm{keV}<\varepsilon_{\mathrm{c}}<100 \mathrm{keV}[6,7]$;

- proportional to either the critical photon energy $\varepsilon_{\mathrm{c}}$ or to the SR power when $\varepsilon_{\mathrm{c}}>100$ $\mathrm{keV}[7]$.

Schematically, this dependence is shown in Figure 2.

\subsection{Gas dynamic model}

The vacuum chamber inside the helical undulator is quite simple: it is a circular tube with inner diameter $d$ and a length $L$ between two pumps with an effective pumping speed $S$. The tube has a vacuum conductance $u$ and, if it is cryogenic or coated with a non-evaporable getter (NEG), may pump with a sticking probability $\alpha$. 


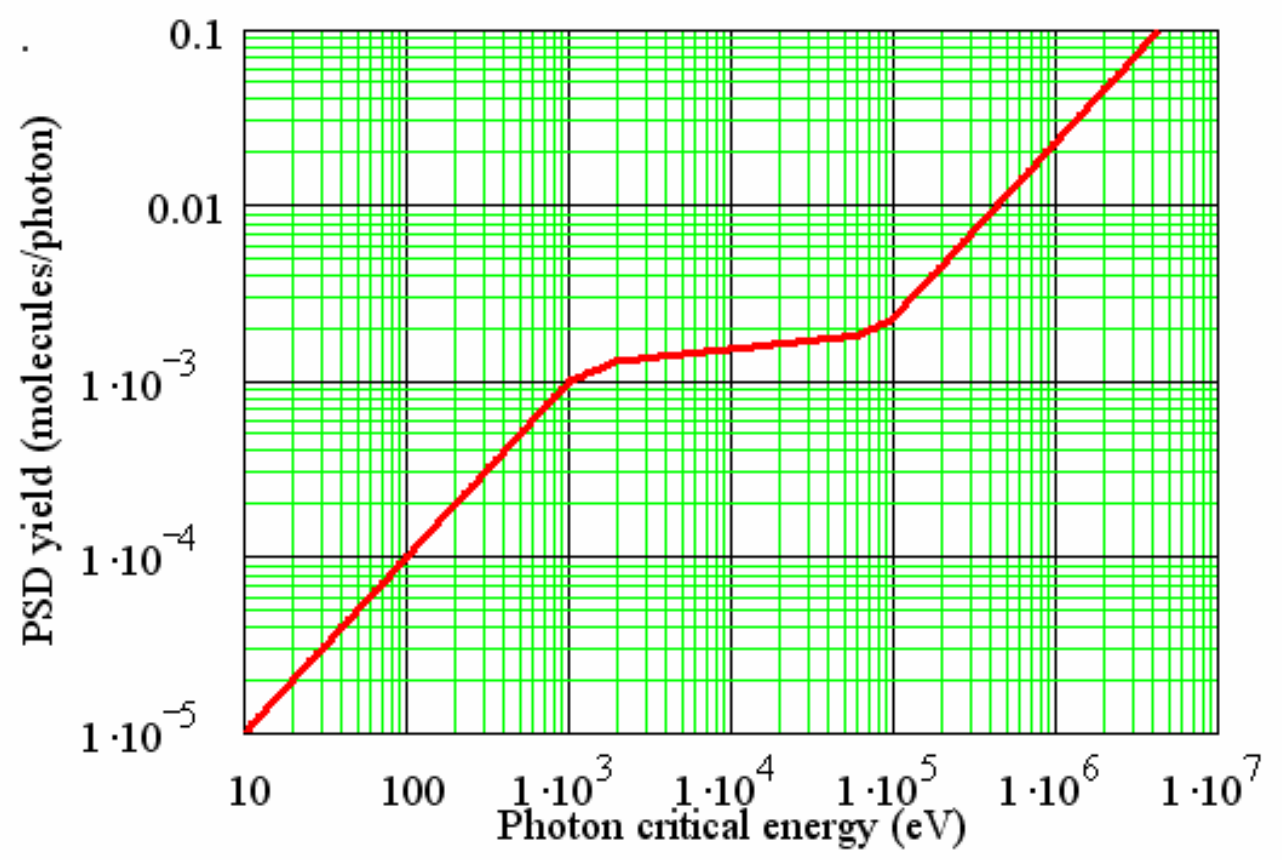

Figure 2. Photon stimulated desorption yield as a function of critical energy of synchrotron radiation from a dipole.

The general equations of gas dynamic balance inside a vacuum chamber with sorbing walls can be written as [8]:

$$
\begin{gathered}
V \frac{d n}{d t}=\left(\eta+\eta^{\prime}(s)\right) \dot{\Gamma}-\alpha C\left(n-n_{e}(s, T)\right)+u \frac{d^{2} n}{d z^{2}} \\
A \frac{d s}{d t}=\alpha C\left(n-n_{e}(s, T)\right)-\eta^{\prime}(s) \dot{\Gamma}
\end{gathered}
$$

where $n\left[\right.$ molecules $\left./ \mathrm{cm}^{3}\right]$ is the volume gas density;

$s$ [molecules $\left./ \mathrm{cm}^{2}\right]$ is the surface density of cryo- or physi-sorbed gas;

$V\left[\mathrm{~cm}^{3}\right]$ is the vacuum chamber volume;

$A\left[\mathrm{~cm}^{2}\right]$ is the vacuum chamber wall area;

$\eta$ [molecules/photon] is the primary photodesorption yield;

$\eta$ ' [molecules/photon] is photodesorption yield of cryo- or physic-sorbed molecules

(so-called secondary photodesorption yield);

$\dot{\Gamma}[$ photon/(sec $\cdot \mathrm{cm})]$ is the photon intensity per unit axial length;

$\alpha$ is the sticking probability;

$C=A \bar{v} / 4$ is the ideal wall pumping speed, $\bar{v}$ is the mean molecular velocity; 
$n_{e}\left[\right.$ molecules $\left./ \mathrm{cm}^{3}\right]$ is the thermal equilibrium gas density;

$u=A_{c} D$ is the specific vacuum chamber conductance per unit axial length, $A_{c}$ is the vacuum chamber cross section; $D$ is the Knudsen diffusion coefficient.

In the quasi-static conditions with $V \frac{\partial n}{\partial t} \approx 0$ and $A \frac{\partial s}{\partial t} \neq 0$ the gas density is described by a second order differential equation for $n(z)$ :

$$
\left(\eta+\eta^{\prime}(s)\right) \dot{\Gamma}-\alpha C\left(n-n_{e}(s, T)\right)+u \frac{d^{2} n}{d z^{2}}=0
$$

coupled to equation (5). For analysis purposes this equation can be re-written in the condensed form:

$$
u \frac{d^{2} n}{d z^{2}}-c n+q=0
$$

where: $c=\alpha C$ is the wall distributed pumping speed;

$q=\left(\eta+\eta^{\prime}(s)\right) \dot{\Gamma}+\alpha C n_{e}(s, T)$ is the (photo- and thermal) desorption.

The second order differential equation (7) for the function $n(z)$ with $u>0, c \geq 0$ and $q \geq 0$ has two solutions:

Case (a)

Case (b)

$$
n(z)=\frac{q}{c}+C_{1} e^{\sqrt{\frac{c}{u}} z}+C_{2} e^{-\sqrt{\frac{c}{u}} z} \text { for } c>0
$$

where the constants depend on the boundary conditions.

In order to study the evolution of the gas density within an accelerator vacuum chamber, the simplest case of the infinitely long tube is studied in the following chapter. Subsequently, the conditions at the ends of the chamber are taken into account and their influence on the gas density analysed. 


\subsubsection{Solutions for an infinitely long vacuum chamber}

The "infinitely long" vacuum chamber approximation can be applied when the pumping effect at the ends is negligible inside the chamber. For an infinitely long vacuum chamber, there is no net axial diffusion. In this case $d^{2} n / d z^{2}=0$ and the solution to equation (7) is

$$
n_{\mathrm{inf}}=\frac{q}{c}=\frac{\left(\eta+\eta^{\prime}(s)\right) \dot{\Gamma}}{\alpha C}+n_{e}(s)
$$

which is independent of the co-ordinate $z$.

The secondary photon stimulated desorption and the thermal equilibrium gas density depends implicitly on the surface density s of cryo- or physi-sorbed molecules. In the case of the cryogenic surface it is:

$$
s(t)=s_{0}+\frac{1}{A} \int_{t=0}^{t} \eta \dot{\Gamma} d t
$$

Hence the gas density inside the cryogenic vacuum chamber will increase with the accumulated photon dose due to photon stimulated desorption. In the case of the NEG coated surface it is different as photons stimulate two processes: desorption and activation of the NEG surface [13], the net effect, whether gas density increases or decreases with time should be studied in future experimentally.

\subsubsection{Short vacuum chamber}

In this paper one refers to "short" vacuum chamber when the conditions at the extremities of the chamber have an influence on the gas density along the whole length of the chamber. In this chapter the different boundary conditions, i.e. known gas density or pumping speed at the ends of the chamber (corresponding to the relevant cases) will be studied.

\subsubsection{Solution for a short vacuum chamber with a given pressure at the ends}

This case can be used for both the operation of the accelerator or in the case of a laboratory experiment. Consider a vacuum chamber centered at $z=0$, of length $L$, and with gas density 
at the ends of $n(-L / 2)=n_{1}$ and $n(L / 2)=n_{2}$. As discussed previously, there are two different solutions for the gas density, depending on the value of the parameter $c$, given by (8)-(9):

Case (a): sorbing walls, $c>0$

$$
C_{1}=\frac{n_{1}+n_{2}-2 n_{\mathrm{inf}}}{4 \cosh \left(\frac{\omega L}{2}\right)}+\frac{n_{2}-n_{1}}{4 \sinh \left(\frac{\omega L}{2}\right)} ; \quad C_{2}=\frac{n_{1}+n_{2}-2 n_{\mathrm{inf}}}{4 \cosh \left(\frac{\omega L}{2}\right)}+\frac{n_{1}-n_{2}}{4 \sinh \left(\frac{\omega L}{2}\right)}
$$

For $n_{1}=n_{2}$ the expression for the gas density may be written as

$$
(z)=n_{\mathrm{inf}}-\left(n_{\mathrm{inf}}-n_{1}\right) \frac{\cosh (\omega z)}{\cosh \left(\frac{\omega L}{2}\right)}
$$

where $n_{\text {inf }}$ is given by equation (7) and $\omega=\sqrt{\alpha C / u}$.

Case (b): non-sorbing walls, $c=0$

The expression for the gas density is:

$$
n(z)=\frac{\left(\eta+\eta^{\prime}\right) \dot{\Gamma}+\alpha C n_{e}}{2 u}\left(\left(\frac{L}{2}\right)^{2}-z^{2}\right)+\frac{n_{1}-n_{2}}{2 L} z+\frac{n_{1}+n_{2}}{2}
$$

This solution is always stable and corresponds to a parabolic pressure profile.

\subsubsection{Solution for a short vacuum chamber with a given pumping speed at the ends}

Consider a vacuum chamber of length $L$ centered at $z=0$, with pumps at the two ends of pumping speed $S$. The conditions at the ends are:

$$
n( \pm L / 2)=\mp \frac{d n( \pm L / 2)}{d z} \frac{u}{S}
$$

In the case (a) when $c>0$ the gas density $n(z)$ is given by: 
EUROTeV-Report-2006-086

$$
n(z)=n_{\text {inf }}\left(1-\frac{\cosh (\omega z)}{\cosh \left(\frac{\omega L}{2}\right)\left(1+\frac{u}{S} \omega \tanh \left(\frac{\omega L}{2}\right)\right)}\right) .
$$

It is useful to calculate the average value of the gas density in the vacuum chamber of length L:

$$
\langle n(L)\rangle=n_{\text {inf }}\left(1-\frac{2 \tanh \left(\frac{\omega L}{2}\right)}{\frac{\omega L}{2}\left(1+\frac{u}{S} \omega \tanh \left(\frac{\omega L}{2}\right)\right)}\right) .
$$

In the case (b) when $c=0$ the gas density $n(z)$ is given by:

$$
n(z)=\left(\left(\eta+\eta^{\prime}\right) \dot{\Gamma}+\alpha C n_{e}\right)\left[\frac{1}{2 u}\left(\left(\frac{L}{2}\right)^{2}-z^{2}\right)+\frac{L}{2 S}\right]
$$

The average value of the gas density in the vacuum chamber of length $L$ in this case is:

$$
\langle n(L)\rangle=\left(\left(\eta+\eta^{\prime}\right) \dot{\Gamma}+\alpha C n_{e}\right)\left(\frac{L}{12 u}+\frac{1}{2 S}\right) L
$$

\subsubsection{Solution for a short vacuum chamber in the equilibrium state}

In the equilibrium state, the condition $A \frac{d s}{d t} \approx 0$ is satisfied, and equation (5) assumes the form:

$$
\alpha C\left(n-n_{e}(s, T)\right)-\eta^{\prime}(s) \dot{\Gamma}=0 .
$$

Then the equation (5) can be simplified as follows:

$$
u \frac{d^{2} n}{d z^{2}}+\eta \dot{\Gamma}=0
$$


In this case the gas density depends only on the primary photon stimulated desorption yields $\eta$, and not on the desorption of cryosorbed molecules, the sticking probability, the surface coverage or the thermal equilibrium gas density. Mathematically, this is a case (b) - solution for a vacuum chamber with non-pumping walls.

\section{Calculations of photon flux and energy irradiating the helical undulator walls}

The parameters we need to know for modelling the gas density along the helical undulator are:

- The photon flux per longitudinal distance

- The desorption yields as a function of

o photon dose

o photon energy

0 vessel material

o temperature

In this part of the report, two characteristics of the photons irradiating the helical undulator vacuum chamber shall be evaluated. All the experimental data on photo-desorption yields is for SR from dipoles or wigglers, where the radiation can be described by the critical energy, photon flux and power, however here the spectrum is from a helical undulator which is significantly different. In a conventional undulator the radiation passes through the undulator vacuum chamber without any collision with the walls. For the ILC helical undulator this is not the case and off-axis radiation can bombard the undulator vacuum chamber, this is mainly due to the long length $L$ and small diameter $d$ giving a large ratio $L / d$.

An estimation of the photon flux onto the vacuum chamber walls for a helical undulator with parameters used in Table 1 was made for a round vacuum chamber with inner diameter ID $=4 \mathrm{~mm}$ (see Figure 4). Only the first hundred meters have been considered for this calculation and the undulator is assumed to be made of $2 \mathrm{~m}$ long modules each acting independently. 
Table 1: Undulator \& Beam Parameters

\begin{tabular}{|l|l|l|}
\hline Energy & $\mathrm{GeV}$ & 150 \\
\hline Current & $\mathrm{A}$ & $45 \cdot 10^{-6}$ \\
\hline Period & $\mathrm{mm}$ & 12 \\
\hline $\mathrm{K}_{\mathrm{x}}, \mathrm{K}_{\mathrm{y}}$ & & $0.84,0.84$ \\
\hline$\sigma_{x}, \sigma_{y}$ & $10^{-6} \mathrm{~m}$ & $66.75,4.45$ \\
\hline$\sigma_{x^{\prime}}, \sigma_{y^{\prime}}$ & $10^{-6} \mathrm{rad}$ & $0.3,4.45$ \\
\hline
\end{tabular}

Two methods had to be used for these calculations. The numeric code SPECTRA [9] was used to calculate the photon spectrum per meter, from which the photon flux and power can be calculated.

Due to the significantly different parameters (such as the undulator length and electron beam energy) of the ILC positron undulator compared to an undulator operating in a light source it was found that at low photon energies, which corresponds to large angles or distances less than $\sim 30 \mathrm{~m}$ from the beginning of an undulator module, SPECTRA was found to be too time consuming on a standard desktop PC for accurate calculation of the spectrum (see the undulator spectrum in Figure at $30 \mathrm{~m}$ to see this effect starting). This effect is not normally noticed because generally such large angles are not important for short undulators typically used in light sources. Therefore only SPECTRA results at distances above $\sim 30 \mathrm{~m}$ from the beginning of the first undulator module have been considered.

The angular distribution of the power from a helical undulator with $\mathrm{N}$ periods can be calculated from the following expression [10]:

where: $\quad x_{n}=\frac{2 K n \gamma \theta}{1+K^{2}+(\gamma \theta)^{2}}$,

$\mathrm{k}_{\mathrm{u}}$ is the wave-number of the undulator period, $k_{u}=\frac{2 \pi}{\lambda_{u}}, \omega_{u}=k_{u} c \sqrt{1-\frac{K^{2}}{\gamma^{2}}}$ and $\mathrm{h}$ is the number of harmonics to be included. This expression assumes a pencil beam and has been used to calculate the photon power dissipated in the vacuum chamber walls. The comparison 
of the total power dissipation at the vessel per meter calculated analytically and with SPECTRA is show in Figure 3. One can see that the discrepancy between the two methods is small for large distances and grows closer to the beginning of undulator. At $\mathrm{z}=30 \mathrm{~m}$ there is almost a factor of 2 difference in the two methods. These discrepancies arise because:

- The analytic expression does not include the beam size or divergence.

- For each point in the SPECTRA output power there is no data for the preceding $30 \mathrm{~m}$ of undulator (as described above).

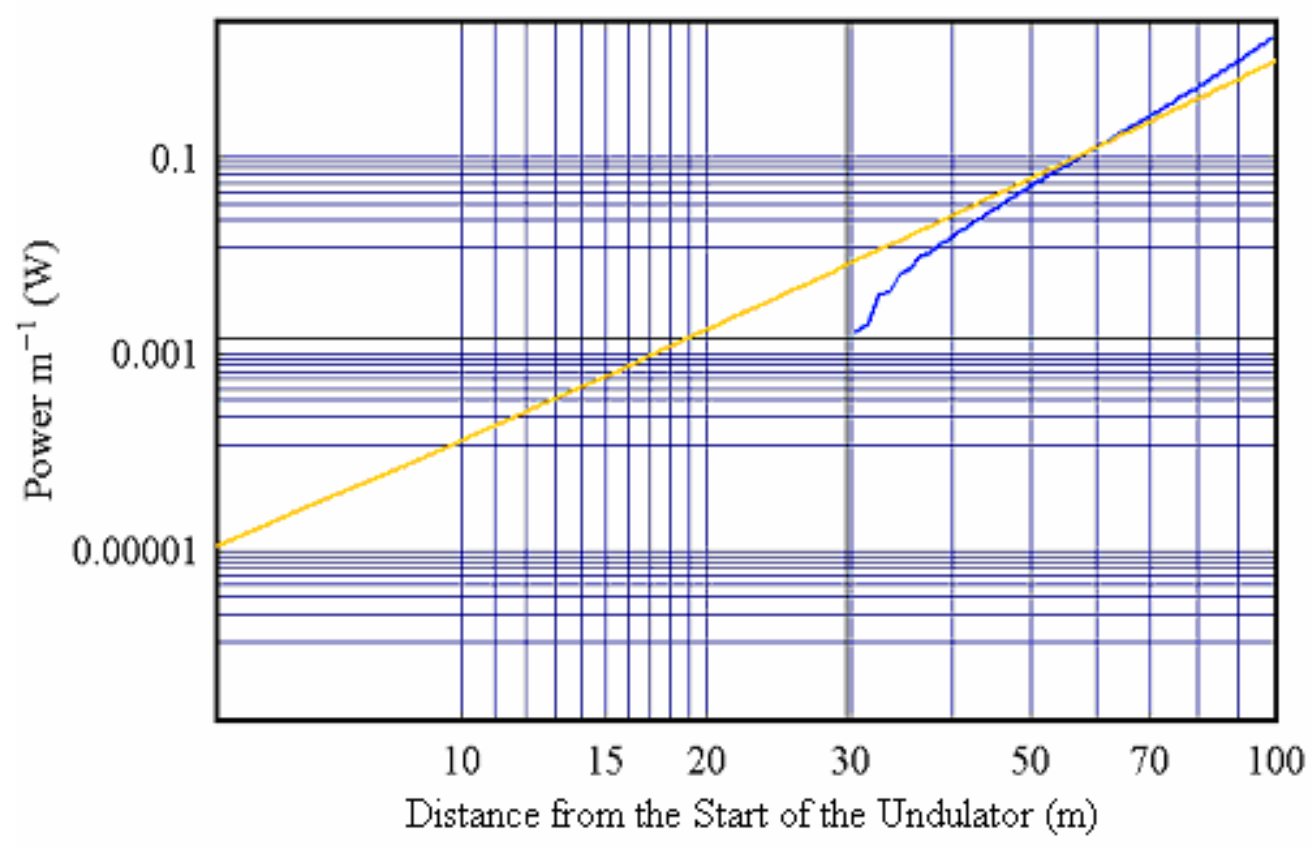

Figure 3: total power deposited in the walls, analytic expression orange, SPECTRA data blue.

The photon flux and spectrum were calculated with SPECTRA for each metre between $30 \mathrm{~m}$ and $100 \mathrm{~m}$ from the beginning of the undulator and are shown in Figure 4 and Figure. It is important to note here that the PSD yields (Figure 2) were measured for SR from a dipole as a function of the critical energy of the dipole radiation (DR). To calculate the PSD due to undulator radiation (UR), which has a different spectrum to that of dipole radiation, the UR (blue line in Figure 4 and Figure) has been approximated by modelling it as a superposition of two separate bending magnet spectra, one with high critical energy (red line in Figure 4 and Figure) and one with low critical energy (green line in Figure 4 and Figure). The critical energy of the DR has been defined as the peak of the undulator flux for the low energy case 
('equivalent critical energy' as shown on Figure 6) and the peak at $\sim 10 \mathrm{MeV}$ (the first harmonic cut-off energy) for the high energy case. The relative power of each DR spectrum was set by assuming that the ratio of the high energy DR power to low energy DR power was the same as that of the power at the peak undulator flux to the power at $\sim 10 \mathrm{MeV}$. The critical energy, $\varepsilon_{c}$, can be calculated (in units of eV) from the energy of the electrons, E, in $\mathrm{GeV}$, and the magnetic field, $\mathrm{B}$, in $\mathrm{T}$, by:

$$
\varepsilon_{c}=665 E^{2} B
$$

The total power, $\mathrm{P}_{\mathrm{t}}$, from a bending magnet, in $\mathrm{kW}$, is:

$$
P_{t}=88.46 \frac{E^{4} I_{b}}{\rho_{0}}
$$

where $I_{b}$ is the bean current, in $A$ and $\rho_{0}$ is the bending radius in $m$. The electron energy is always assumed to be $150 \mathrm{GeV}$. The difference in photon intensity (or number of photons per meter per second) between DR and UR in Figure 4 can be explained by the fact that SPECTRA has not been used to accurately calculate photons with energy less then $\sim 100 \mathrm{keV}$, (due to limitation in computing power). This is clearly seen on Figure 5. 


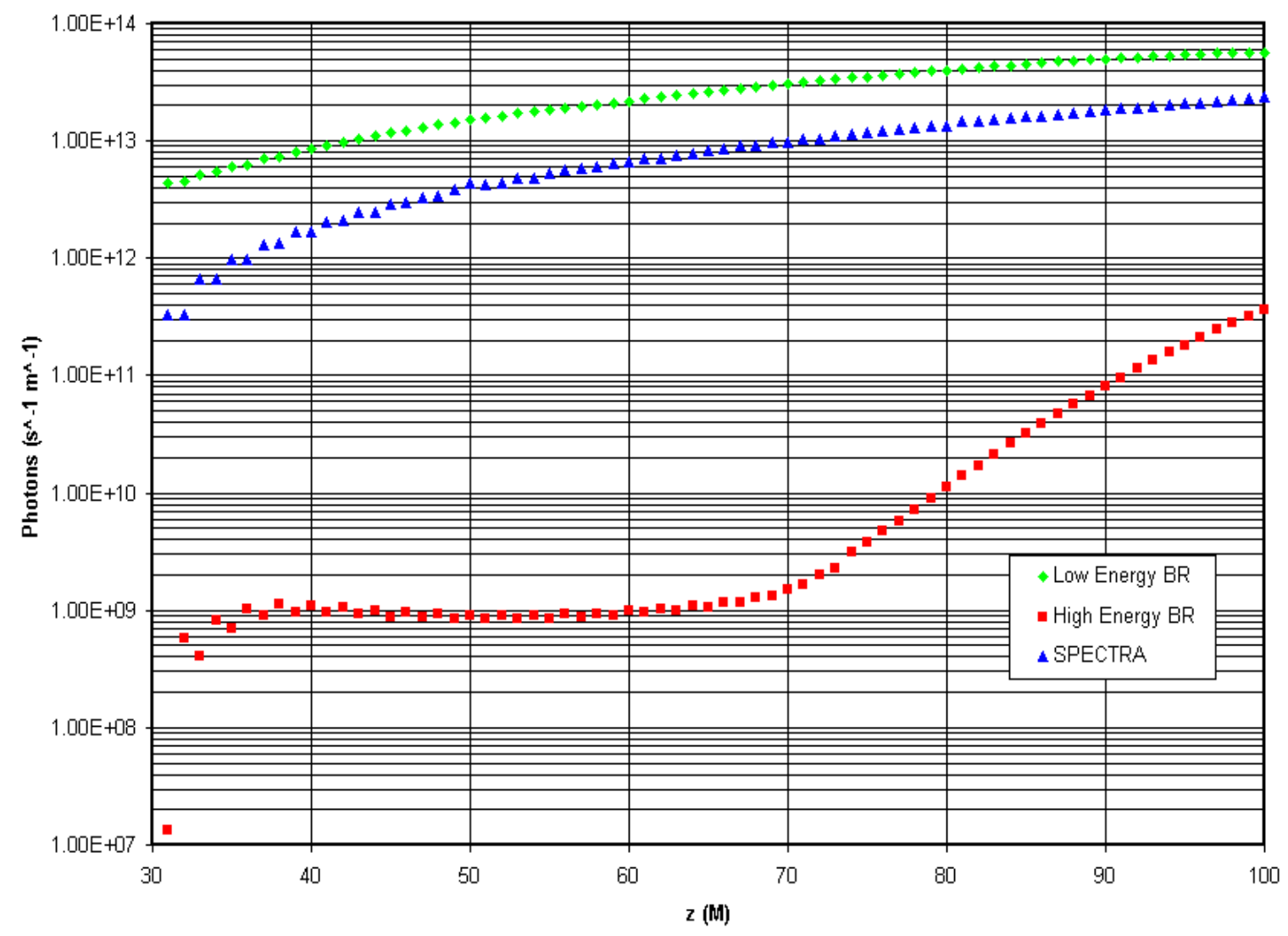

Figure 4: Photons flux onto the helical undulator vacuum chamber walls for the three models.

The photon energy spectrum from the undulator and from the dipole approximations is shown in Figure at four different places: 30, 40, 50 and 100 meters downstream of beginning of the undulator. Figure 4 and Figure show that near the start of the undulator it is the low energy photons that dominate but further down the device the higher energy photons become more significant, as expected. There is a fairly good agreement between the SPECTRA data and the DR. The main differences can be seen at lower photon energies, which is expected due to the assumptions made and the limitations of the SPECTRA code outlined above. Figure 6 shows how the peak of the SPECTRA flux changes with distance along the undulator. Equivalent critical energy was calculated from the analytically calculated power. It matches well with the peak energy of the SPECTRA for $40 \mathrm{~m}<\mathrm{z}<100 \mathrm{~m}$, therefore it was used for photon flux calculation for $5 \mathrm{~m}<\mathrm{z}<40 \mathrm{~m}$. 

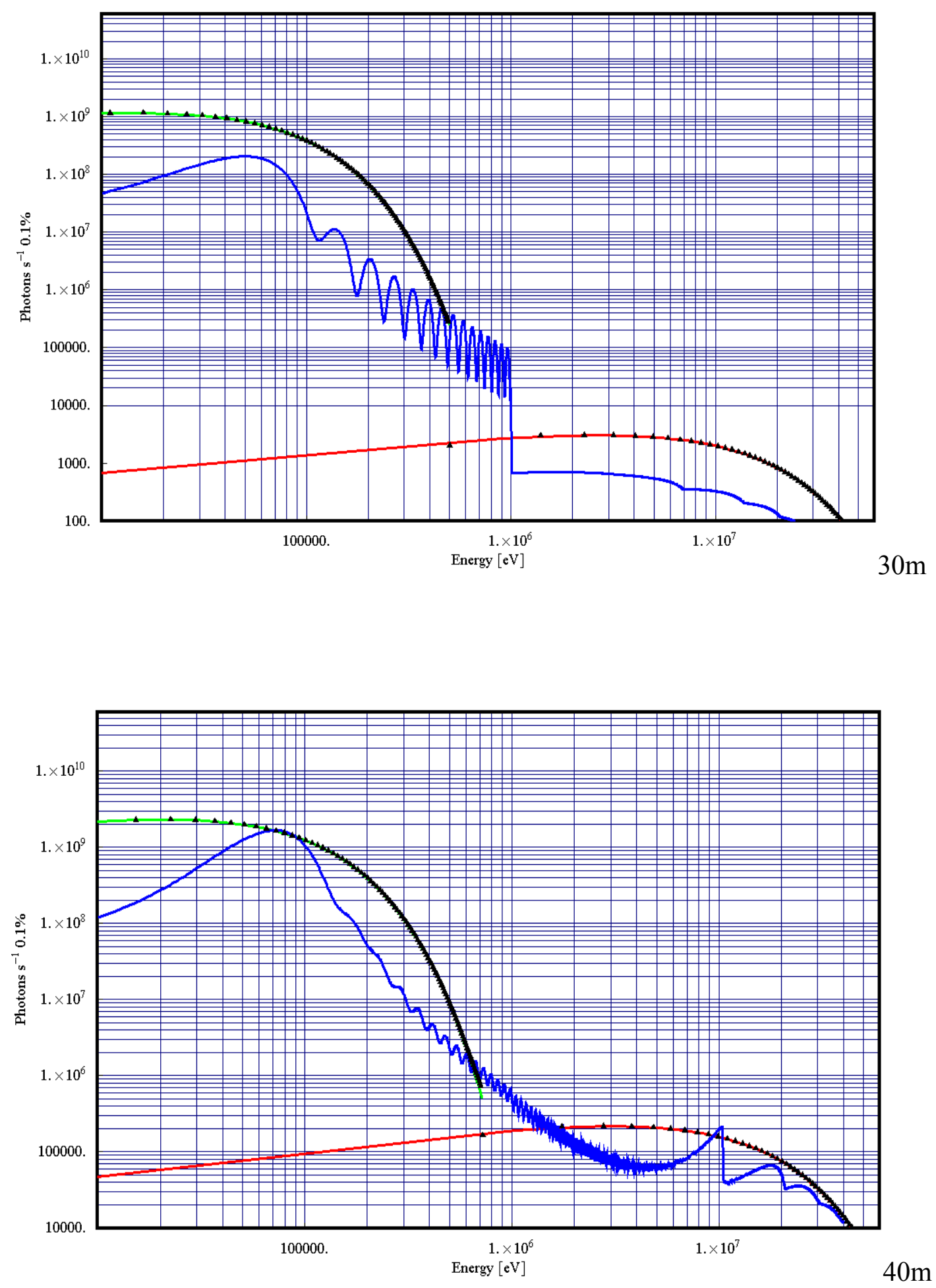

Figure 5 (a). Energy of photons hitting the undulator vacuum chamber walls in 4 different places: SPECTRA data (blue) vs dipole radiation: $\varepsilon_{c}=E_{p}$ (green) and $\varepsilon_{c} \sim 10 \mathrm{MeV}$ (red) and the superposition of the two dipole radiation spectra (points). 

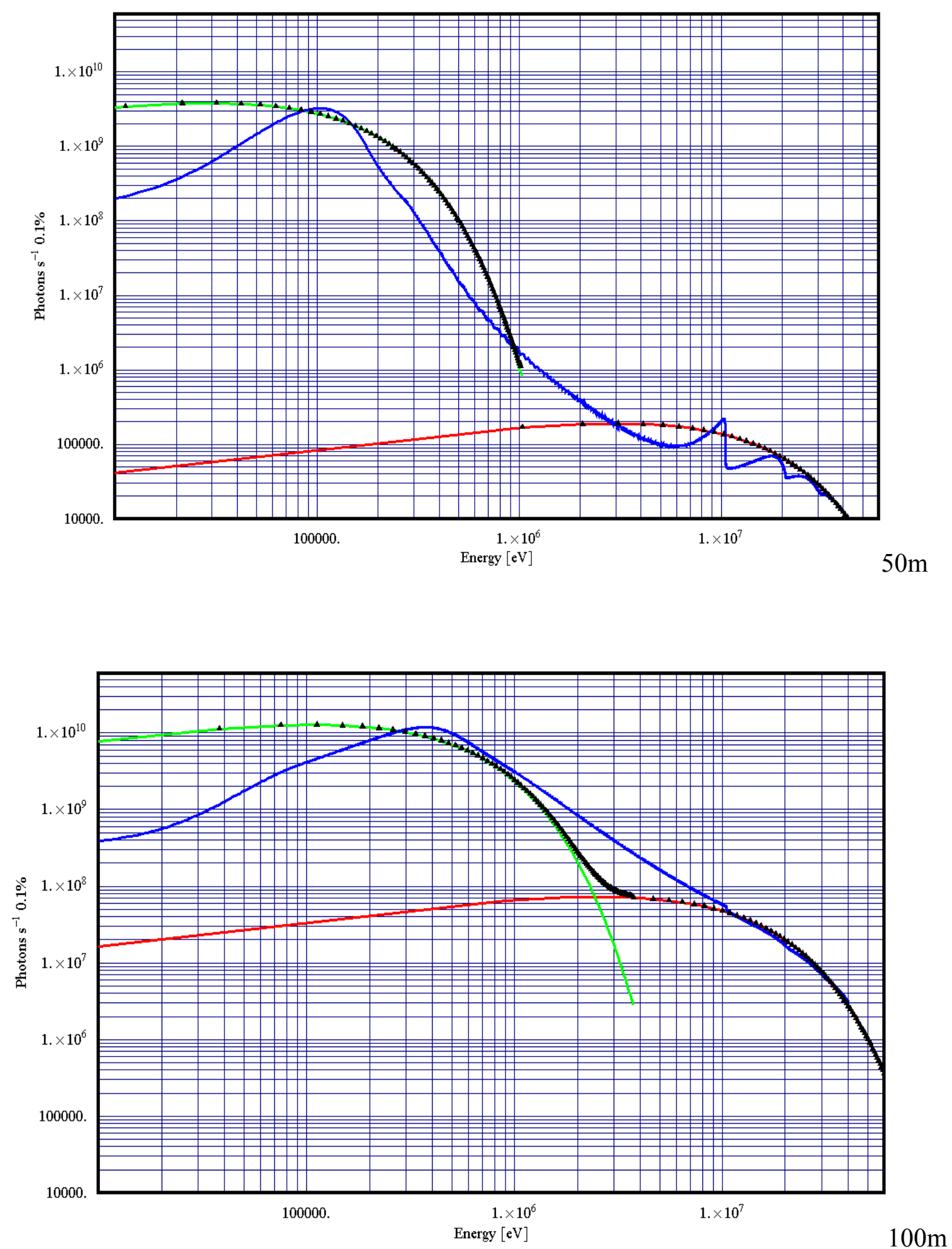

Figure 5 (b). Energy of photons hitting the undulator vacuum chamber walls in 4 different places: SPECTRA data (blue) vs dipole radiation: $\varepsilon_{\mathrm{c}}=\mathrm{E}_{\mathrm{p}}$ (green) and $\varepsilon_{\mathrm{c}} \sim 10 \mathrm{MeV}$ (red) and the superposition of the two dipole radiation spectra (points). 


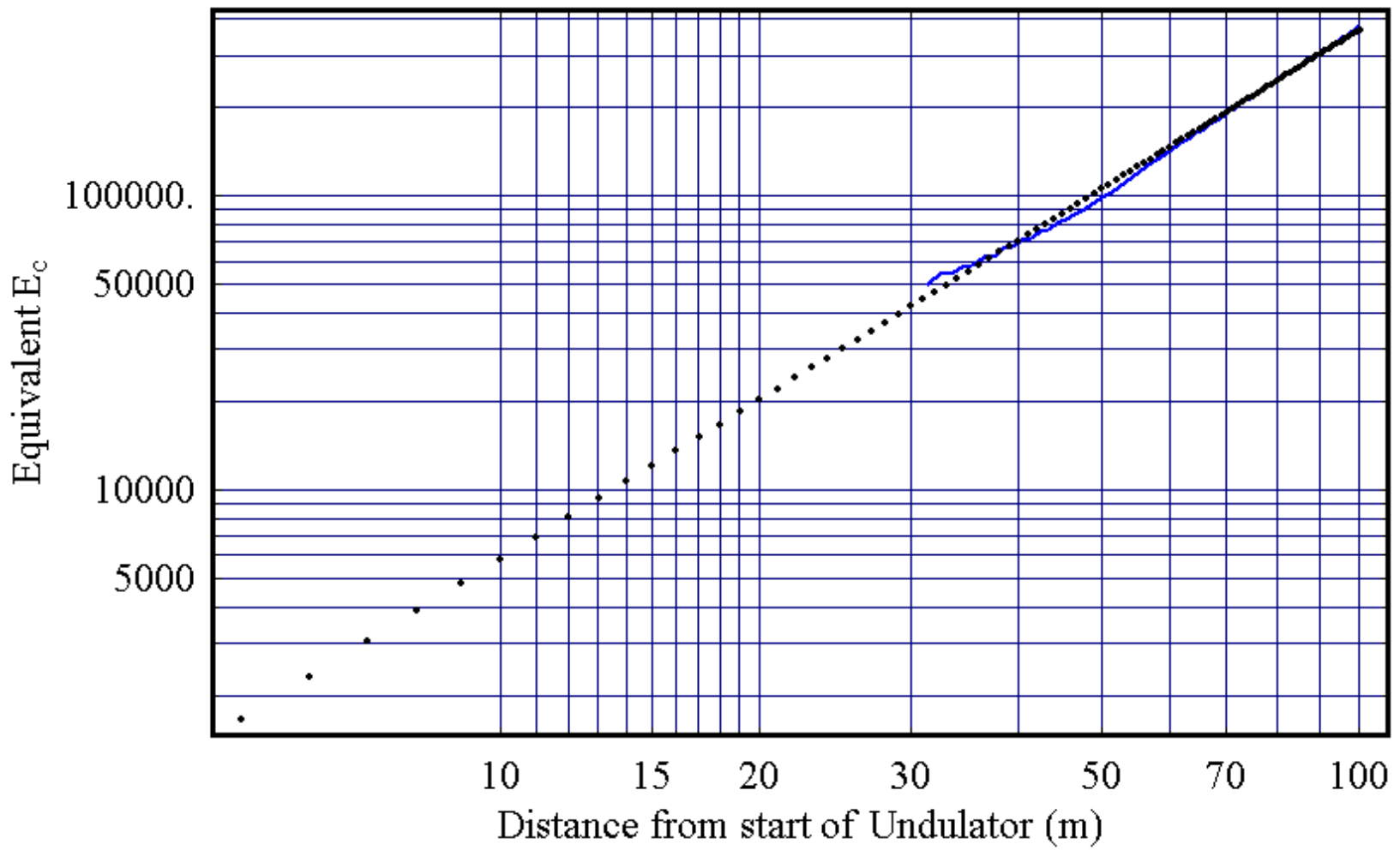

Figure 6. Equivalent critical energy of photons (points) and peak energy of photons calculated from peak of SPECTRA data (blue line) as a function of the distance from the start of the undulator.

\section{Gas density calculations for Helical Undulator.}

The gas density along the helical undulator is limited by the fast ions instability calculated by L. Wang to be 100 nTorr [11], which corresponds to a gas density of $3.3 \cdot 10^{15}$ molecules $/ \mathrm{m}^{3}$. The highest gas density along the vacuum chamber will be reached at the end of the undulator and can be estimated assuming the following:

- The photon flux is $\Gamma=2.3 \cdot 10^{13}$ photons $/ \mathrm{m}$ at $\mathrm{z}=100 \mathrm{~m}$;

- Maximum equivalent critical energy is $400 \mathrm{keV}$;

- Since there is no experimental data for desorption yields of different materials for such a high energy the desorption yield at $\varepsilon_{\mathrm{c}}=400 \mathrm{keV}$ can be estimated as the following: 
- At room temperature there are experimental data for the desorption yields for different materials measured at $\varepsilon_{\mathrm{c}}=3 \mathrm{keV}[12,13]$, than using Figure 2 one can write:

$$
\eta\left(\varepsilon_{\mathrm{c}}=400 \mathrm{keV}\right) \approx 6 \eta\left(\varepsilon_{\mathrm{c}}=3 \mathrm{keV}\right)
$$

- At $4.2 \mathrm{~K}$ the only available experimental data for the desorption yields for different materials were measured at $\varepsilon_{\mathrm{c}}=300 \mathrm{eV}$ [5], in this case:

$$
\eta\left(\varepsilon_{\mathrm{c}}=400 \mathrm{keV}\right) \approx 30 \eta\left(\varepsilon_{\mathrm{c}}=300 \mathrm{eV}\right)
$$

In this case the photon stimulated desorption is calculated as: $q=\eta \dot{\Gamma}$. Desorption yields are shown in Table 2. These are the highest values, desorption reduces with an accumulated photon dose.

\begin{tabular}{|c|c|c|c|c|c|c|c|c|}
\hline \multirow{2}{*}{$\begin{array}{l}\text { Material } \\
\varepsilon_{\mathrm{c}}(\mathrm{keV})\end{array}$} & \multicolumn{2}{|c|}{$\begin{array}{l}\text { Aluminium } \\
\text { at } \mathrm{T}=300 \mathrm{~K}\end{array}$} & \multicolumn{2}{|c|}{$\begin{array}{l}\text { Stainless steel } \\
\text { at } \mathrm{T}=300 \mathrm{~K}\end{array}$} & \multicolumn{2}{|c|}{$\begin{array}{l}\text { Copper coated } \\
\text { stainless steel }\end{array}$} & \multicolumn{2}{|c|}{ NEG coated SS } \\
\hline & 3 & 400 & 3 & 400 & 0.3 & 400 & 4.5 & 400 \\
\hline \multicolumn{9}{|c|}{ Initial desorption yields } \\
\hline $\mathrm{H}_{2}$ & 0.05 & 0.3 & $1.2 \cdot 10^{-3}$ & $7.2 \cdot 10^{-2}$ & $3.5 \cdot 10^{-3}$ & $2.1 \cdot 10^{-2}$ & $1.5 \cdot 10^{-5}$ & $9 \cdot 10^{-5}$ \\
\hline $\mathrm{CH}_{4}$ & $2 \cdot 10^{-3}$ & 0.012 & $5 \cdot 10^{-5}$ & $3 \cdot 10^{-4}$ & $1 \cdot 10^{-6}$ & $3 \cdot 10^{-5}$ & $2 \cdot 10^{-7}$ & $1.2 \cdot 10^{-6}$ \\
\hline $\mathrm{CO}$ & 0.025 & 0.15 & $3 \cdot 10^{-4}$ & $1.8 \cdot 10^{-3}$ & $2 \cdot 10^{-4}$ & $6 \cdot 10^{-4}$ & $1 \cdot 10^{-5}$ & $6 \cdot 10^{-5}$ \\
\hline $\mathrm{CO}_{2}$ & 0.012 & 0.072 & $2 \cdot 10^{-4}$ & $1.2 \cdot 10^{-3}$ & $2 \cdot 10^{-5}$ & $6 \cdot 10^{-5}$ & $2 \cdot 10^{-6}$ & $1.2 \cdot 10^{-5}$ \\
\hline \multicolumn{9}{|c|}{ Desorption yields after about 100 days of SR conditioning at $\mathrm{z}=100 \mathrm{~m}$} \\
\hline $\mathrm{H}_{2}$ & $3 \cdot 10^{-3}$ & 0.018 & $3 \cdot 10^{-4}$ & $1.8 \cdot 10^{-3}$ & $4 \cdot 10^{-4}$ & $1.2 \cdot 10^{-3}$ & $1.5 \cdot 10^{-5}$ & $9 \cdot 10^{-5}$ \\
\hline $\mathrm{CH}_{4}$ & $8 \cdot 10^{-6}$ & $5 \cdot 10^{-5}$ & $5 \cdot 10^{-6}$ & $3 \cdot 10^{-5}$ & $2 \cdot 10^{-7}$ & $6 \cdot 10^{-7}$ & $2 \cdot 10^{-7}$ & $1.2 \cdot 10^{-6}$ \\
\hline $\mathrm{CO}$ & $6 \cdot 10^{-4}$ & $4 \cdot 10^{-3}$ & $5 \cdot 10^{-5}$ & $3 \cdot 10^{-4}$ & $8 \cdot 10^{-5}$ & $2.4 \cdot 10^{-4}$ & $1 \cdot 10^{-5}$ & $6 \cdot 10^{-5}$ \\
\hline $\mathrm{CO}_{2}$ & $8 \cdot 10^{-4}$ & $5 \cdot 10^{-3}$ & $3 \cdot 10^{-5}$ & $1.8 \cdot 10^{-4}$ & $4 \cdot 10^{-6}$ & $1.2 \cdot 10^{-5}$ & $2 \cdot 10^{-6}$ & $1.2 \cdot 10^{-5}$ \\
\hline
\end{tabular}

Table 2. Desorption yields for Al and SS. 
Thermal stimulated desorption must be also considered in cases of aluminium and stainless steel and is the lowest limit. After the bakeout it can be in the range of $10^{-12} \mathrm{mbar} \cdot 1 \mathrm{t} /\left(\mathrm{s} \cdot \mathrm{cm}^{2}\right)$ at room temperature. Thermal stimulated desorption from the NEG is negligible; it is certainly less then $10^{-13}$ mbar. Gas density at room temperature was calculated for the layout as shown in Figure 7.

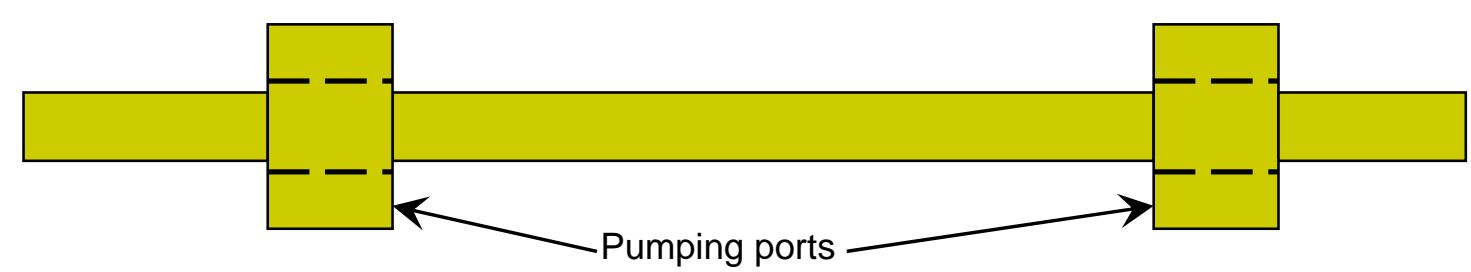

Figure 7. Room temperature vacuum chamber layout.

\subsection{Gas density along the stainless steel tube}

Stainless steel is one of the best conventional materials used for accelerator vacuum chambers, after bakeout at $300^{\circ} \mathrm{C}$ for $24 \mathrm{hrs}$ it provides the lowest photon stimulated desorption. The gas density along the stainless steel tube irradiated by photons with parameters corresponding $\mathrm{z}=100 \mathrm{~m}$ is shown on Figure 8 as a function of distance between the vacuum pumps, photon stimulated desorption was calculated for four gas species, $\mathrm{H}_{2}$, $\mathrm{CH}_{4}, \mathrm{Co}$ and $\mathrm{CO}_{2}$, and the thermal desorption for $\mathrm{CO}$ with $\eta_{\mathrm{t}}=10^{-12} \mathrm{mbar} \cdot 1 /\left(\mathrm{s} \cdot \mathrm{cm}^{2}\right)$. The dotted line shows the required gas density of $3.3 \cdot 10^{15}$ molecules $/ \mathrm{m}^{3}$ (100 nTorr at room temperature).

One can see that the required gas density will be achieved after 100 days beam conditioning for the stainless steel vacuum chamber with a vacuum pump every 6 metres. 

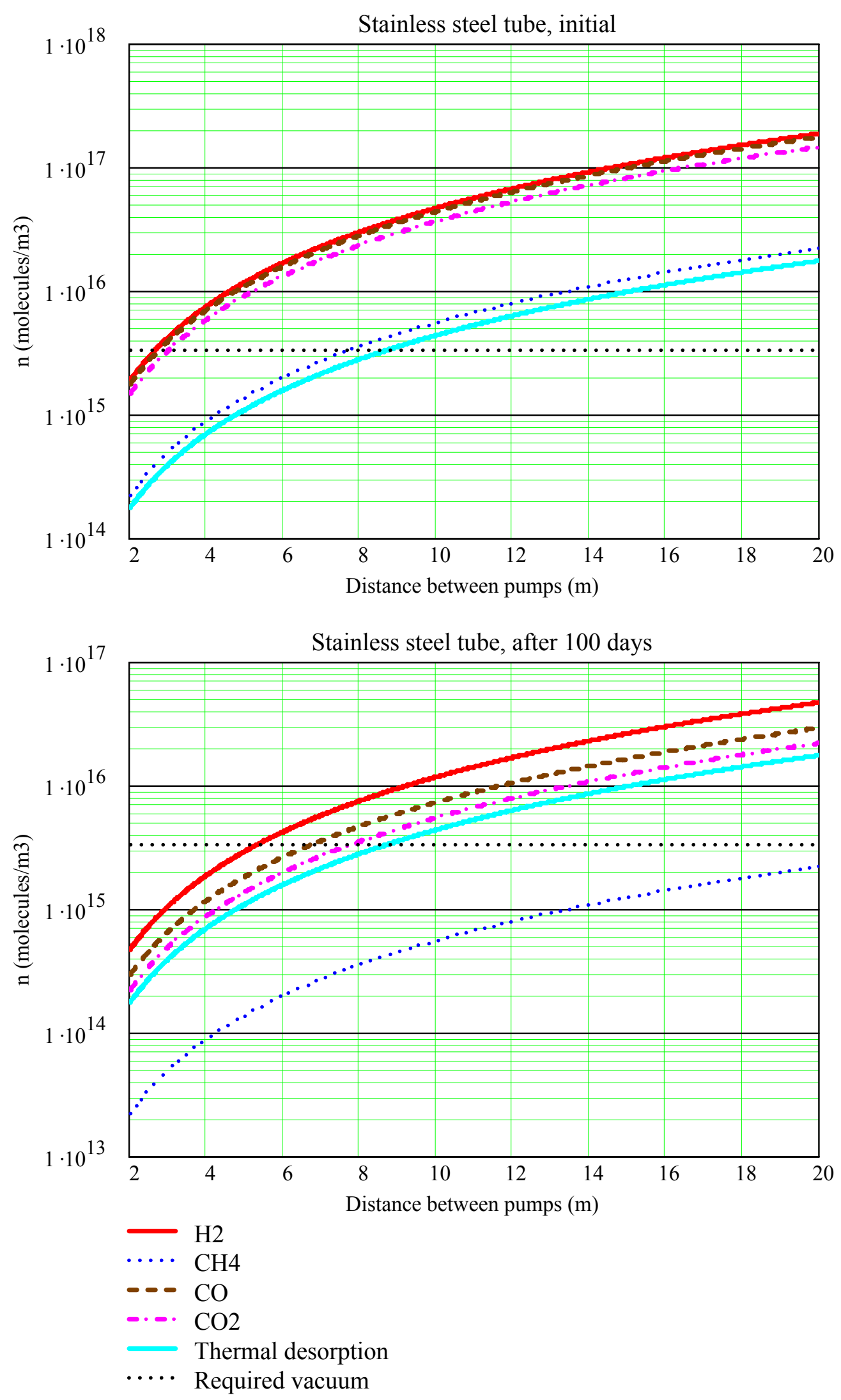

Figure 8. Gas density in stainless steel vacuum chamber near $z=100 \mathrm{~m}$, initial and after 100 day of conditioning with a beam. No SR collimation. 


\subsection{Gas density for the NEG coated tube}

A new vacuum technology for accelerators, recently developed at CERN, is a TiZrV coating for all inner area of vacuum chamber. This coating plays a double role: it reduces the outgassing from the vacuum chamber walls and it introduces the distributed pumping speed, resulting in lower gas density [13]. The only gases which are not pumped by such a coating are hydrocarbons and noble gases. The use of this coating requires activation, i.e. 24 hours bakeout at $180^{\circ} \mathrm{C}$. This conditioning effect would be negligible for the ILC undulator. Results of gas density calculations are shown on Figure 9.

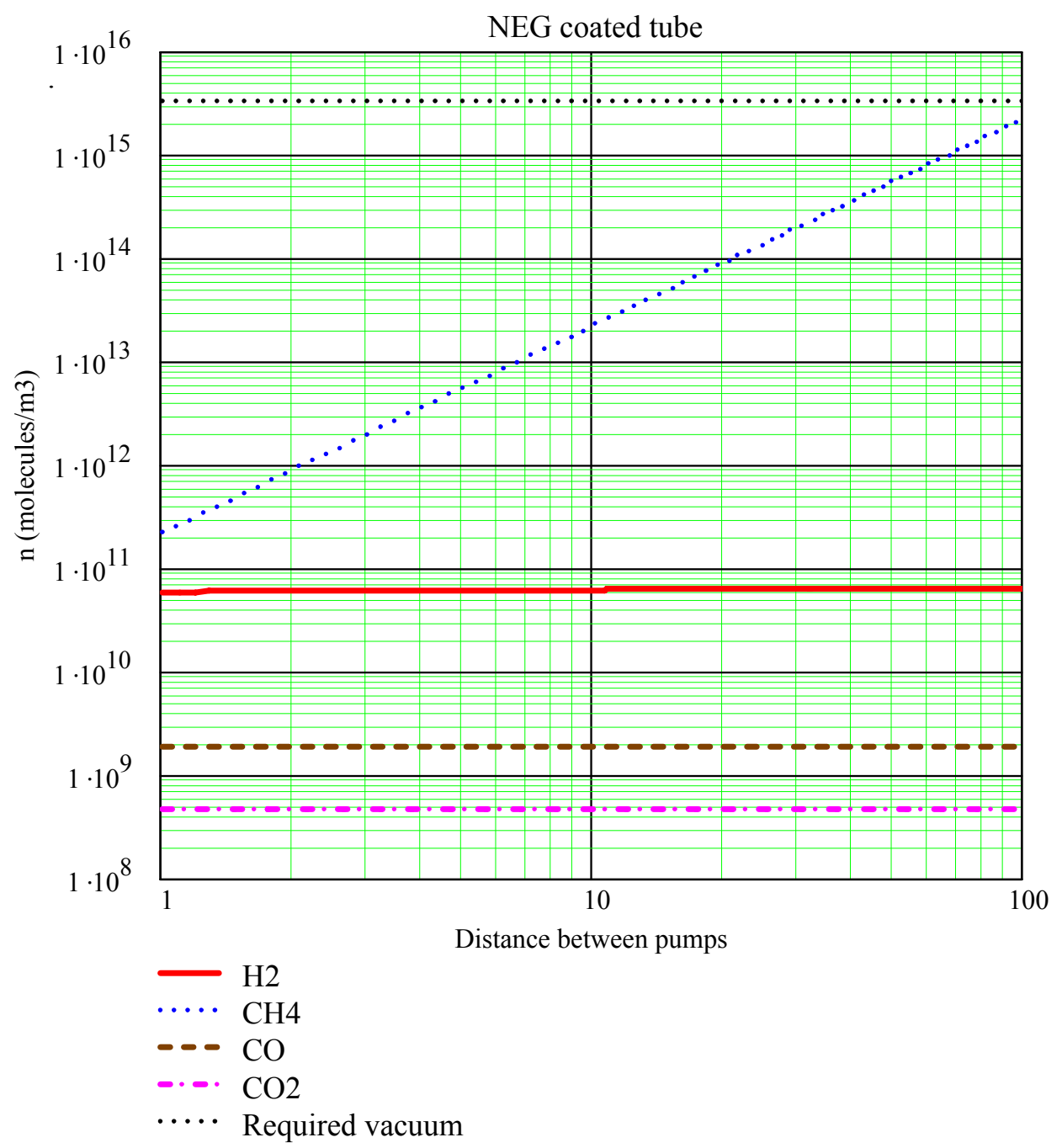

Figure 9. Gas density in TiZrV NEG coated vacuum chamber near $\mathrm{z}=100 \mathrm{~m}$. No SR collimation. 
One can see that gas density in the TiZrV NEG coated vacuum chamber does not require many external pumps for pumping the gas desorbed from the NEG, calculations shows that it required to install a pump every 50-100 $\mathrm{m}$. In this case the pumps will be needed to pump any potential leaks, because even a small leak to atmosphere may cause the increase of Ar gas density, therefore it would be generally beneficial to install a small pump (like Starcell or triode SIP with pumping speed of $\sim 201 / \mathrm{s}$ ) every $15-20 \mathrm{~m}$.

\subsection{Gas density for copper coated stainless steel tube at $4.2 \mathrm{~K}$}

In the case of the superconducting undulator the vacuum chamber will be at cryogenic temperature. The gas dynamic in the vacuum chamber at cryogenic temperature is well studied [5]. Initially the gas density is defined by photon stimulated desorption of molecules from the subsurface layers of vacuum chamber. Then the desorbed molecules are cryosorbed on the surface with lower bounding energy which led to two effects:

- The photon stimulated desorption of cryosorbed molecules with much higher desorption yields;

- The vapour equilibrium gas density increases.

Both effects cause the gas density to increase to the level limited by the end pumping

The gas density at cryogenic temperature was calculated for the layout as shown on Figure 10, and the results are shown on Figure 11.

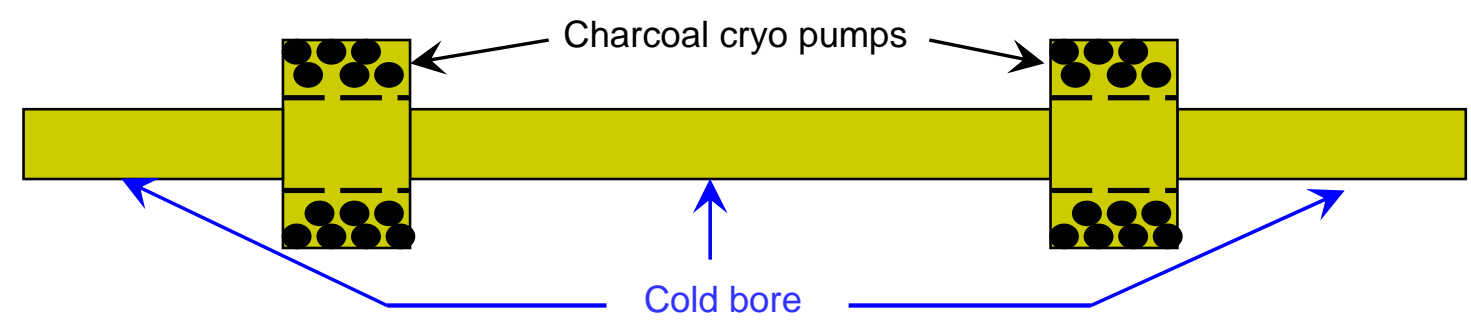

Figure 10. Cryogenic vacuum chamber layout. 

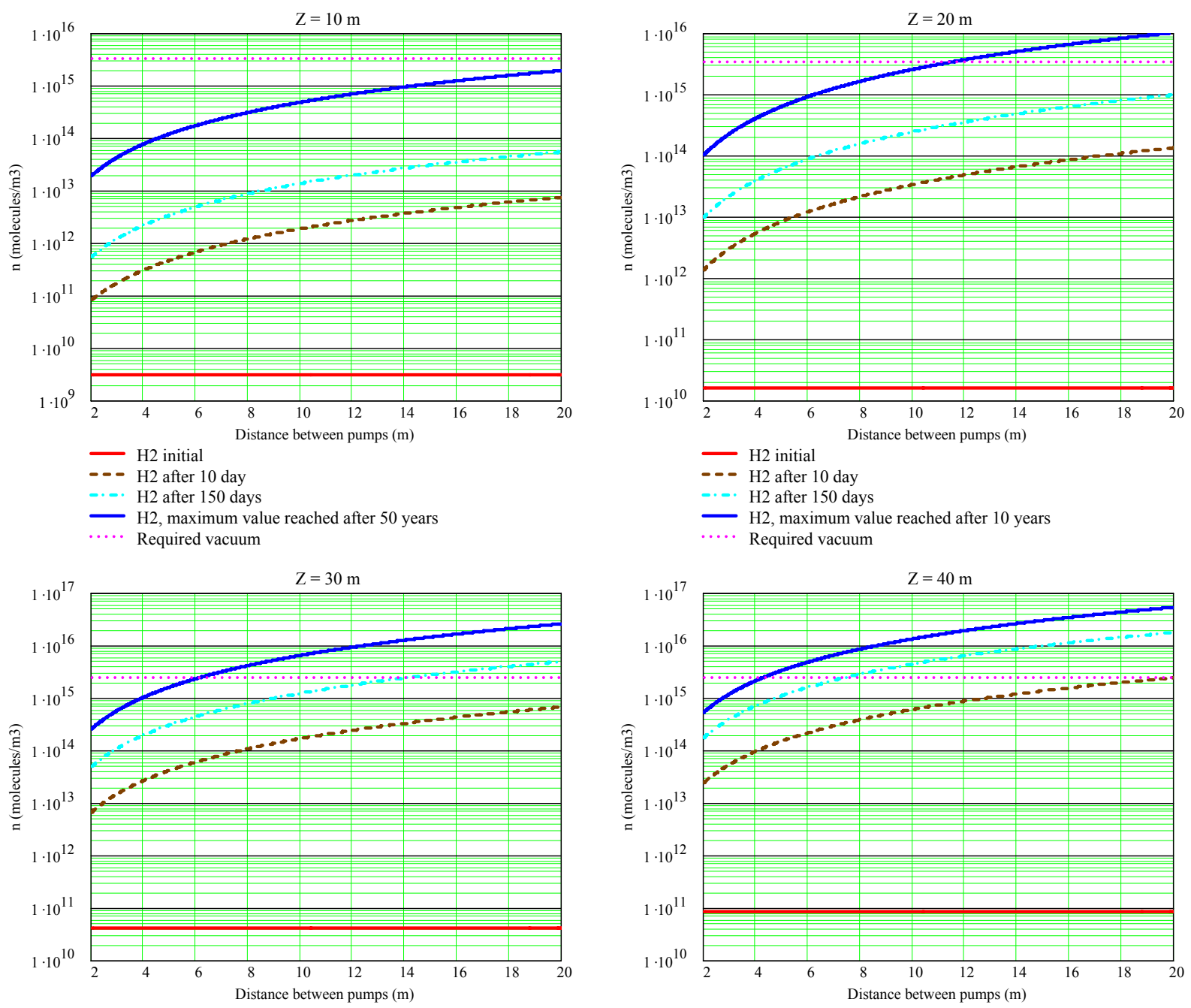

- $\mathrm{H} 2$ initial

-- H2 after 10 day

-. H2 after 150 days

- H2, maximum value reached after 4 years Required vacuum

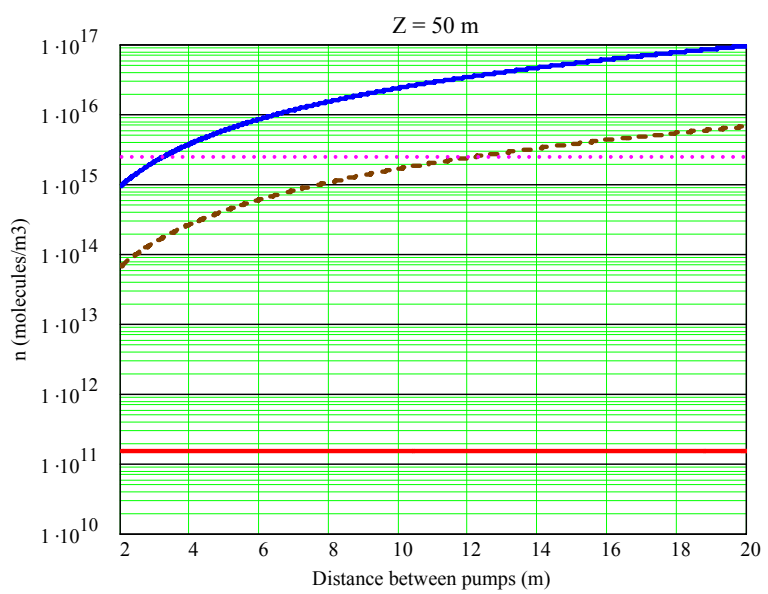

- $\mathrm{H} 2$ initial

-.- H2 after 10 day

-. H2 after 150 day

- $\mathrm{H} 2$, maximum value reached after 2 years Required vacuum

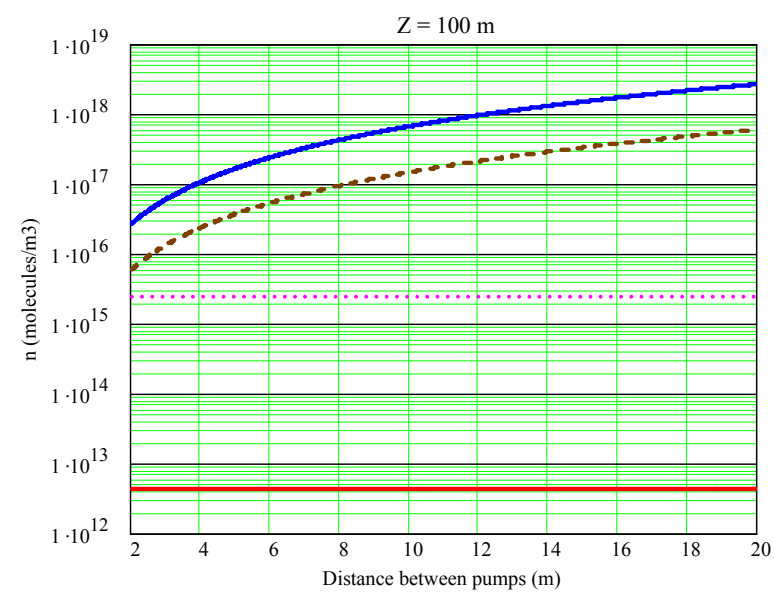

$$
\begin{array}{ll}
- & \text { H2 initial } \\
\ldots & \text { H2 after } 10 \text { day } \\
\ldots & \text { H2, maximum value reached after } 1 \text { year } \\
\ldots \cdots & \text { Requred vacuum }
\end{array}
$$

-.- H2 after 1 day

H2, maximum value reached after 10 days - Requred vacuum

Figure 11. Gas density in cryogenic vacuum chamber near $\mathrm{z}=100 \mathrm{~m}$. No SR collimation. 
Although the initial gas density is much lower than the required level, the photo desorbed gas will condense on the tube walls. The $\mathrm{H}_{2}$ gas density will increase with time due to photodesorption of cryosorbed gas from initial (red line) to maximum (blue line). The gas density after 10 and 150 days of irradiation are shown as dashed brown and dash-dot cyan lines, respectively. The vacuum design should allow the continuous operation of the undulator for at least a half a year without warming up, which would be necessary for pumping away the condensed $\mathrm{H}_{2}$ and reducing the $\mathrm{H}_{2}$ dynamic gas density. Results of calculations are summarised in Table 3, the criteria of continuous operation for at least 150 days was studied to meet another criteria of the reasonable distance between pumps, which was assumed to be about $10-20 \mathrm{~m}$. The main conclusion is that these two criteria are met for distances from the beginning of the undulator of $\mathrm{z} \leq 30 \mathrm{~m}$.

Table 3. Required distance between pumps to keep the gas density inside the cryogenic vacuum chamber below the required limit.

\begin{tabular}{|l|c|c|}
\hline \multirow{2}{*}{$\begin{array}{l}\text { Distance from } \\
\text { the beginning of the }\end{array}$} & \multicolumn{2}{|c|}{ Distance between pumps } \\
\cline { 2 - 3 } & $\begin{array}{c}\text { gas density always below } \\
\text { the required limit }\end{array}$ & $\begin{array}{c}\text { gas density below the } \\
\text { required limit during } \\
\text { for about 150 days }\end{array}$ \\
\hline$Z=10 \mathrm{~m}$ & $\leq \mathbf{2 0} \mathrm{m}$ & $>\mathbf{2 0} \mathrm{m}$ \\
\hline$Z=20 \mathrm{~m}$ & $\leq \mathbf{1 0} \mathrm{m}$ & $\leq \mathbf{2 0} \mathrm{m}$ \\
\hline$Z=30 \mathrm{~m}$ & $\leq 6 \mathrm{~m}$ & $\leq 7.5 \mathrm{~m}$ \\
\hline$Z=40 \mathrm{~m}$ & $\leq 4 \mathrm{~m}$ & $\leq 4.5 \mathrm{~m}$ \\
\hline$Z=50 \mathrm{~m}$ & $\leq 3 \mathrm{~m}$ & - \\
\hline$Z=100 \mathrm{~m}$ & - & - \\
\hline
\end{tabular}

That means that to keep gas density on the acceptable level the SR beam collimators must be installed in a few location along the undulator vacuum chamber. Collimators will be a section of vacuum chamber with reduced aperture, combined with a pumping port, something similar to that shown in Figure 12. The distance between collimators depends on the ratio of the 
diameters of collimator to vacuum chamber. Simple geometry calculations show that the distance between collimators, $\mathrm{L}_{\mathfrak{c}}$, should be:

- $\mathrm{L}_{\mathrm{c}} \leq 15 \mathrm{~m}$ for $\mathrm{d}_{\mathrm{c}}: \mathrm{d}=1: 2$

- $\mathrm{L}_{\mathrm{c}} \leq 7.5 \mathrm{~m}$ for $\mathrm{d}_{\mathrm{c}}: \mathrm{d}=3: 4$

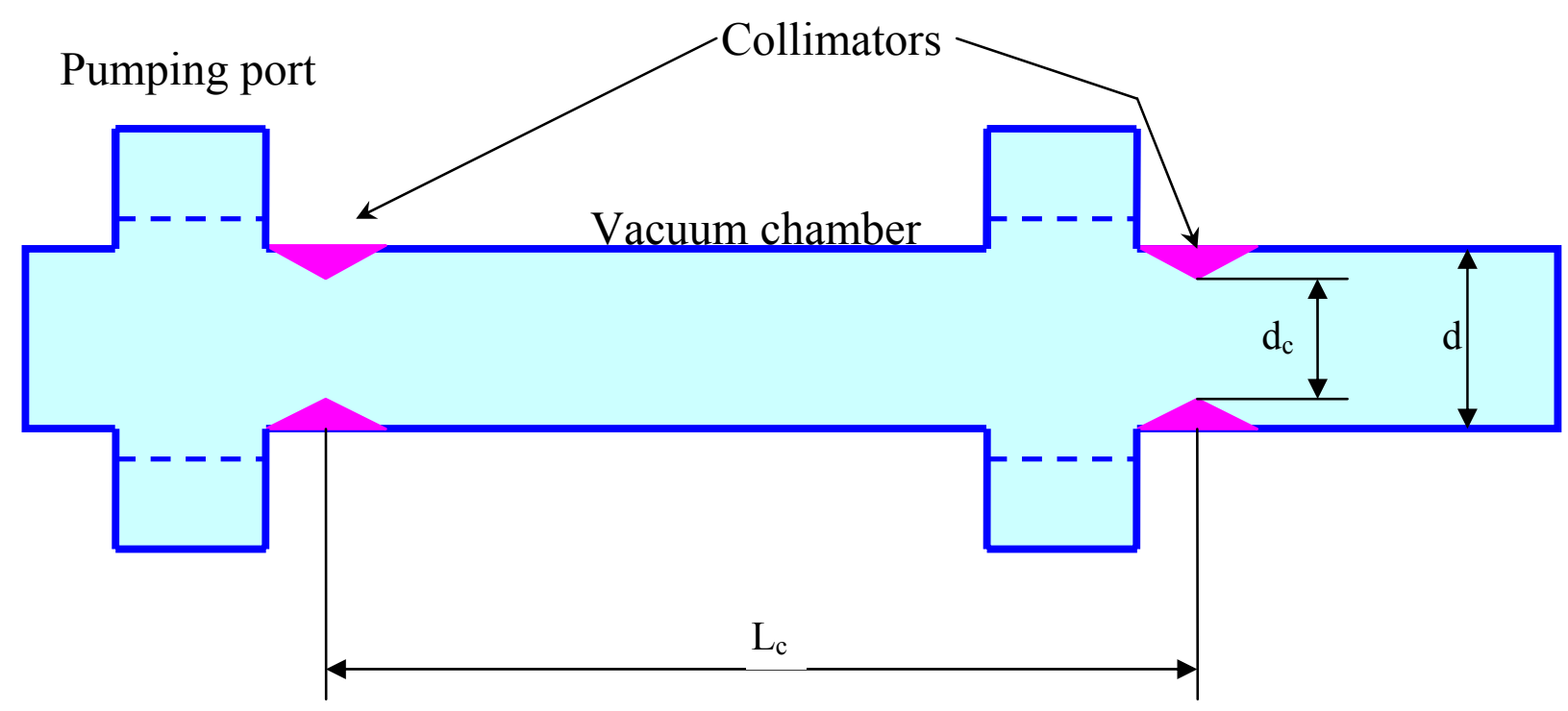

Figure 12. Layout of collimators in vacuum chamber.

It is possible that a pure copper pipe could be used. If a steel pipe is used it would need to be coated in a high conductivity metal to reduce wakefield effects. The coating of such a pipe is not trivial and for this reason a copper pipe might be used. If another material is used it is worth remembering that:

- Presently there are no experimental results for other materials measured at $4.2 \mathrm{~K}$.

- Stainless steel without copper coating is expected to have lower desorption yield, hence gas density will be less, but the impedance might be too high.

- Aluminium is a better conductor than stainless steel, but desorption yields at room temperature are higher than for stainless steel with or without copper coating:

$\mathrm{O}$ it looks reasonable to expect higher desorption of aluminium at $4.2 \mathrm{~K}$;

o surface anodisation may help [14], further experimental studies are required. 
- Use of the NEG coating might be beneficial for reducing desorption yields of all used materials, although no additional pumping is expected. Further experimental studies are needed with NEG coated vacuum chamber irradiated at $4.2 \mathrm{~K}$.

\section{Conclusions \& Further Work}

The vacuum requirement for the undulator can be fulfilled but the choice of technical solution depends on a number of unknowns:

- Cryogenic or RT vacuum chamber

- Stainless steel, copper or aluminium alloy vacuum chamber

- High conductance coating of vacuum chamber if necessary: copper or gold

- There are many uncertainties in inputs for vacuum calculations:

o Desorption yields of different materials irradiated by photon with energies from $30 \mathrm{keV}$ to $30 \mathrm{MeV}$ (lack of experimental data)

o Calculation of flux and energy of photons irradiating the undulator vacuum chamber

o Therefore a safety margin is essential.

- RT vacuum chamber must be NEG coated.

o Investments and studies in these technologies

- Cryogenic vacuum chamber:

o SS walls are preferable,

o Copper walls are acceptable,

$\mathrm{O}$ If $\mathrm{Al}$ is required then the inner surface anodisation may be a solution

o SR beam mask (or typed vacuum chamber) is essential every 7.5-15 m.

The full length of the ILC positron undulator could be up to $200 \mathrm{~m}$ for polarised positron production. For this case further studies will have to be carried out to asses the radiation in a longer device as a longer device means that there are more photons that could potentially be incident on the vessel walls. For this work an ID of $4 \mathrm{~mm}$ for the undulator was assumed however the current assumed aperture for the ILC is $5.6 \mathrm{~mm}$, this increase in aperture will reduce the number of photons incident on the vessel walls and should be included in future calculations. 


\section{Acknowledgments}

This work is supported by the Commission of the European Communities under the $6^{\text {th }}$ Framework Programme "Structuring the European Research Area", contract number RIDS011899.

\section{References}

[1] C. Herbeaux, P. Martin, V. Baglin and O. Gröbner. "Photon stimulated desorption of an unbaked stainless steel chamber by $3.75 \mathrm{keV}$ critical energy photons." J.Vac.Sci.Technol A17(2), Mar/Apr 1999, pp.635-643.

[2] C.L. Foerster, H. Halama and C. Lanni. "Photon-stimulated desorption yields from stainless steel and copper-plated beam tubes with various pre-treatments." J.Vac.Sci.Technol A8(3), May/June 1990, pp.2856-2859.

[3] O. Gröbner, A.G. Mathewson and P. Martin. "Gas desorption from an oxygen free high conductivity copper vacuum chamber by synchrotron radiation photons." J.Vac.Sci.Technol A12(3), May/Jun 1994, pp.846-853.

[4] R. Calder, O. Gröbner, A.G. Mathewson, V.V. Anashin, A. Dranichnikov, O. Malyshev. Synchrotron radiation induced gas desorption from a prototype Large Hadron Collider beam screen at cryogenic temperatures. J. Vac. Sci. Technol. A 14(4), pp. 2618-2623, Jul/Aug 1996.

[5] V.V. Anashin, G.E. Derevyankin, V.G. Dudnikov, O.B. Malyshev, V.N. Osipov, C.L. Foerster, F.M. Jacobsen, M.W. Ruckman, M. Strongin, R. Kersevan, I.L. Maslennikov, W.C. Turner and W.A. Landford. Cold beam tube photodesorption and related experiments for SSCL $20 \mathrm{TeV}$ proton collider. J. Vac. Sci. Technol. A 12(4), pp. 16631672, Jul/Aug 1994. (SSCL-Preprint-533, Nov. 1993).

[6] J. Gomez-Goni, O. Gröbner and A.G. Mathewson. Comparison of photodesorption yields using synchrotron radiation of low critical energies for stainless steel, copper and electropolished copper surfaces. J.Vac.Sci.Technol. A 12(4), pp. 1714-1718, Jul/Aug 1994.

[7] J.C. Billy, J.P. Bojon, B Henrist, N. Hilleret, M.J. Jimrnrz, I. Laugier, P. Stubin. The pressure and gas composition evolution during the operation of the LEP accelerator at 100 GeV. Vacuum 60, 2001, pp. 183-189.

[8] V.V. Anashin, O.B. Malyshev, V.N. Osipov, I.L. Maslennikov and W.C. Turner. "Investigation of synchrotron radiation-induced photodesorption in cryosorbing quasiclosed geometry", J.Vac.Sci.Technol. A 12(5), pp. 2917-2921, Sep/Oct 1994.

[9] T. Tanaka and H. Kitamura "SPECTRA: a Synchrotron Radiation Calculation Code," J.Synch.Rad. (2001) 8, pages 1221-1228

[10] B.M. Kincaid, "A Short Period Helical Wiggler as an Improved Source of Synchrotron Radiation,” J.Appl.Phys. Vol. 48, No 7, 1977 Pages 2684-2691

[11] L. Wang. Fast ion instability at undulator insert of the ILC, March 2006, SLAC

[12] A.G. Mathewson, O. Gröbner, P. Strubin, P. Martin, R. Souchet. Comparison of Synchrotron Radiation induced gas desorption fron $\mathrm{Al}$, stainless steel and $\mathrm{Cu}$ vacuum chamber. CERN/AT-VA/90-31, CERN, Geneva, 1990. 
[13] V.V. Anashin, I.R. Collins, R.V. Dostovalov, N.V. Fedorov, A.A. Krasnov, O.B. Malyshev and V.L. Ruzinov. Comparative study of photodesorption from TiZrV coated and uncoated stainless steel vacuum chambers. Vacuum 75 (2), July 2004, pp. 155-159.

[14] V.V. Anashin, R.V. Dostovalov, A.A. Krasnov, I.R. Collins, O.B. Malyshev, A.A. Nikiforov, V.A. Cherepanov, Z.A.Korotaeva, A.E.Lapin, V.A.Poluboyarov. Molecular cryosorption properties of porous copper, anodised aluminium and charcoal at temperatures between 10 and 20 K. Vacuum 76(1), October 2004, pp. 23-29. 